\title{
Erratum to: Multipliers of Embedded Discs
}

\author{
Kenneth R. Davidson • Michael Hartz • \\ Orr Moshe Shalit
}

\begin{abstract}
In the originally published article, there is a gap that resulted from relying on a result that appeared in the literature but was false. In this note we point to the mistake and describe the consequences. With some additional care, our main results are shown to hold.
\end{abstract}

\section{Erratum to: Complex Anal. Oper. Theory DOI 10.1007/s11785-014-0360-8}

\section{Statement of the Gap and Consequences}

In the originally published article, there is a gap in the proof of Theorem 2.4 where we invoke Proposition 3.2 in an early version of [3]. This result says that the characters of $\mathcal{M}_{V_{1}}$ lying in $\pi^{-1}\left(\mathbb{B}_{d}\right)$ are precisely point evaluations. The proof of the cited Proposition, in turn, relied on [1, Theorem 3.2], which states that the characters of $\mathfrak{L}_{d}$ lying in $\pi^{-1}\left(\mathbb{B}_{d}\right)$ are precisely point evaluations. That theorem is not true for $d=\infty$, as the following example shows. In the example, we work with the algebra $\mathcal{M}_{\infty}$ of multipliers on Drury-Arveson space.

The online version of the original article can be found under doi:10.1007/s11785-014-0360-8.

K. R. Davidson · M. Hartz

Department of Pure Mathematics, University of Waterloo, Waterloo, ON, Canada

e-mail: krdavids@uwaterloo.ca

\section{Hartz}

e-mail:mphartz@uwaterloo.ca

O. M. Shalit $(\varangle)$

Department of Mathematics, Ben-Gurion University of the Negev, Beersheva, Israel e-mail: oshalit@math.bgu.ac.il; oshalit@bgu.ac.il 
Example Let $\left(v_{n}\right)$ be a sequence in $\mathbb{B}_{\infty}$ with the property that $\left\|v_{n}\right\| \rightarrow 1$, but $\left(v_{n}\right)$ converges weakly to zero. By passing to a subsequence, we may assume that $\left(v_{n}\right)$ is interpolating for $\mathcal{M}_{\infty}$ [In the originally published article, Proposition 9.1]. Thus, the unital homomorphism $\Phi: \mathcal{M}_{\infty} \rightarrow \ell^{\infty}$ defined by $\Phi(f)(n)=f\left(v_{n}\right)$ is surjective, so its adjoint $\Phi^{*}$ is an embedding of the Stone-Čech compactification $\beta \mathbb{N}$ into the character space of $\mathcal{M}_{\infty}$. We claim that every point in $\beta \mathbb{N} \backslash \mathbb{N}$ lies in the fiber over the origin, i.e., $\pi\left(\Phi^{*}(\beta N \backslash N)\right)=\{0\}$. Indeed, let $\varphi \in \beta \mathbb{N} \backslash \mathbb{N}$. Then for every $k \geq 1$, we have

$$
\left(\Phi^{*}(\varphi)\right)\left(M_{z_{k}}\right)=\varphi\left(\left(Z_{k}\left(v_{n}\right)\right)\right)=\lim _{n \rightarrow \infty} Z_{k}\left(v_{n}\right)=0
$$

This shows that there are points in $\pi^{-1}\left(\mathbb{B}_{d}\right)$ which are not point evaluations.

We can use this construction to show that there are also algebras $\mathcal{M}_{V}$ with characters that are fibered over points in $\mathbb{B}_{d} \backslash V$. Let $\left(v_{n}\right)$ be as above, and assume that $v_{0}=0$. Let $f \in \mathcal{M}_{\infty}$ satisfy $f(0)=1$ and $f\left(v_{n}\right)=0$ for $n \geq 1$. Then $V=f^{-1}(0)$ is a variety such that $0 \notin V$, but the fiber $\pi^{-1}(0)$ is large.

As a consequence of this problem, our proof of Theorem 2.4, and therefore Corollaries 2.6 and 2.7, are incomplete in the case where $d=\infty$ (we do not know if the result stated is false). This gap does not affect any of the results in Sections 3-6, since they did not require these results in the case $d=\infty$.

We will prove below that Theorem 2.4 (and therefore its corollaries) holds at least when the embedding $f_{1}: \mathbb{D} \rightarrow \mathbb{B}_{\infty}$ is the embedding giving rise to the spaces $\mathcal{H}_{s}$ $(s \leq 0)$ studied in Examples 7.7 and 8.6, which were the cases of greatest interest in the paper. As a result, all the results of Section 7 hold with the exception of the second sentence in the statement of Proposition 7.3, which says that $\mathcal{M}_{V}$ and $\mathcal{M}_{\tilde{V}}$ are isomorphic via the natural map if and only if they are isomorphic via any isomorphism. This statement is still true if one assumes that at least one of the spaces is $\mathcal{H}_{s}(s \in$ $[-1,0])$. Most of the results in Section 8 also hold, with the exception of Lemma 8.4 and statements (1) and (3) in Theorem 8.5. Statements (1) and (3) are not known to hold in full generality, but do hold if one assumes that one of the spaces is $\mathcal{H}_{s}, s<-1$, so Example 8.6 still exhibits an uncountable family of non-isomorphic multiplier algebras associated to compact varieties. Lemma 8.4 holds if the compact variety $\bar{V}$ arises from one of the spaces $\mathcal{H}_{s}, s<-1$, by the results in Section 9 of [4]. As a result, Proposition 9.3 is only known to hold if the compact variety is of this type. The other results in Section 9 are not affected.

\section{Proof of Theorem 2.4 for the Spaces $\mathcal{H}_{s}$}

Recall the statement of Theorem 2.4:

Theorem 2.4 (In the originally published article) Let $V_{i}$ be discs in $\mathbb{B}_{d}$ as described above. Let $\varphi: \mathcal{M}_{V_{1}} \rightarrow \mathcal{M}_{V_{2}}$ be a continuous algebra homomorphism. Then $F=$ $\left.F_{\varphi}\right|_{V_{2}}$ is a holomorphic map with multiplier coefficients. If $F$ is not constant, then $F$ maps $V_{2}$ into $V_{1}$. In this case, $\left.\varphi^{*}\right|_{V_{2}}=F$ and $\varphi$ is given by composition with $F$, that is, 


$$
\varphi(h)=h \circ F \text { for all } h \in \mathcal{M}_{V_{1}} \text {. }
$$

In particular, if $\varphi$ is injective, then $F$ is not constant. And if $\varphi$ is an isomorphism, $F$ is a biholomorphism of $V_{2}$ onto $V_{1}$.

The only problem with the published proof is that there is gap in showing that $\varphi^{*}\left(\rho_{v}\right)$ has to be point evaluation. The proof of the theorem holds for any algebra $\mathcal{M}_{f(\mathbb{D})}$ for which it is true that

(1) for every $\lambda \in V$, the fiber $\pi^{-1}(\lambda)$ is a singleton containing only point evaluation at $\lambda$, and

(2) $\pi\left(M\left(\mathcal{M}_{V}\right)\right) \cap \mathbb{B}_{d}=V$.

If we add the assumption that both algebras $\mathcal{M}_{V_{1}}$ and $\mathcal{M}_{V_{2}}$ have these properties, then the proof as it appears is correct. In Sect. 2.1 we will show that the embeddings considered in Examples 7.7 and 8.6 satisfy properties (1) and (2).

If we only assume that $\mathcal{M}_{V_{1}}$ satisfies properties (1) and (2), then the proof of Theorem 2.4 is only good up to the point where it is proved that if $\varphi$ is an isomorphism then $F$ is a biholomorphism. In the paper we deduced this statement by replacing $\varphi$ with $\varphi^{-1}$. However, if we only assume that $\mathcal{M}_{V_{1}}$ satisfies those properties, then $\varphi$ and $\varphi^{-1}$ are not interchangeable. In Sect. 2.2 below we show how to complete the proof if one only makes special assumptions on $\mathcal{M}_{V_{1}}$.

If $V$ is the intersection of zero sets of functions in $\mathcal{A}_{d}$, the norm closure of the polynomials in $\mathcal{M}_{d}$, then each $f \in \mathcal{A}_{d}$ extends to a continuous function on $\overline{\mathbb{B}_{d}}$. So we can talk about their zero sets in the closed ball.

Proposition Suppose that a variety $V$ in $\mathbb{B}_{d}$ is the intersection of zero sets of a family $\mathcal{F} \subset \mathcal{A}_{d}$ such that $\bigcap_{f \in \mathcal{F}} f^{-1}(0)=\bar{V}$. Then $\pi\left(M\left(\mathcal{M}_{V}\right)\right)=\bar{V}$.

Proof Since $\mathcal{M}_{V} \simeq \mathcal{M}_{d} / J_{V}$ where $J_{V}$ is the ideal of multipliers vanishing on $V$, every character $\varphi$ of $\mathcal{M}_{V}$ lifts to a character $\psi$ of $\mathcal{M}_{d}$ that annihilates $J_{V}$. Assume that $\varphi \in \pi^{-1}(\lambda)$ for $\lambda \in \overline{\mathbb{B}_{d}}$, whence $\psi \in \pi^{-1}(\lambda)$ also. Then $\psi(f)=f(\lambda)$ for every polynomial $f$, and hence for every $f \in \mathcal{A}_{d}$. In particular, as every $f \in \mathcal{F}$ belongs to $J_{V}$, we have $0=\psi(f)=f(\lambda)$. Therefore $\lambda \in \bar{V}$ by hypothesis.

\subsection{Proof that Mult $\mathcal{H}_{s}$ Satisfies Properties (1) and (2)}

The algebras $\mathcal{M}_{V}$ considered in Sections 7 and 8 are determined by the family of polynomials $b_{n} Z_{1}^{n}=b_{1}^{n} Z_{n}$ for $n \geq 2$. It is clear that the intersection of their zero sets in $\overline{\mathbb{B}}_{\infty}$ is exactly $\bar{V}$. Thus they satisfy property (2) by the Proposition.

The following lemma provides a simple sufficient condition under which $\pi^{-1}(0)$ is a singleton.

Lemma In the setting of Sections 7 and 8, the following assertions are equivalent:

(i) For every $g \in \mathcal{M}_{V}$ with $g(0)=0$, there is $\widetilde{g} \in \mathcal{M}_{V}$ such that $g=z_{1} \widetilde{g}$.

(ii) For every $g \in \operatorname{Mult}\left(\mathcal{H}_{f}\right)$ with $g(0)=0$, we have $g / z \in \operatorname{Mult}\left(\mathcal{H}_{f}\right)$.

(iii) The sequence $\left(\frac{a_{n}}{a_{n-1}}\right)_{n \geq 1}$ is bounded. 
Proof The equivalence of (i) and (ii) follows by an application of the isomorphism

$$
\mathcal{M}_{V} \rightarrow \operatorname{Mult}\left(\mathcal{H}_{f}\right), \quad g \mapsto g \circ f
$$

Suppose that (iii) holds. Then

$$
D: \mathcal{H}_{f} \rightarrow \mathcal{H}_{f}, \quad h \mapsto \frac{h-h(0)}{z},
$$

is a bounded linear map. Indeed $D$ maps $z^{n}$ to $z^{n-1}$, and $\left\|z^{n}\right\|^{2}=\frac{1}{a_{n}}$. Let $g \in$ $\operatorname{Mult}\left(\mathcal{H}_{f}\right)$ with $g(0)=0$. Then for every $h \in \mathcal{H}_{f}$, we have

$$
D M_{g} h=D(g h)=\frac{g}{z} h .
$$

This shows that $g / z \in \operatorname{Mult}\left(\mathcal{H}_{f}\right)$ and that $D M_{g}=M_{g / z}$. Hence, (ii) holds.

Conversely, suppose that (ii) is satisfied. Then

$$
\widetilde{D}: \operatorname{Mult}\left(\mathcal{H}_{f}\right) \rightarrow \operatorname{Mult}\left(\mathcal{H}_{f}\right), \quad g \mapsto \frac{g-g(0)}{z},
$$

is defined and clearly linear. Since convergence in $\operatorname{Mult}\left(\mathcal{H}_{f}\right)$ implies pointwise convergence on $\mathbb{D}$, we conclude with the help of the closed graph theorem that $\widetilde{D}$ is bounded. In particular,

$$
\frac{1}{a_{n-1}}=\left\|z^{n-1}\right\|_{\operatorname{Mult}\left(\mathcal{H}_{f}\right)}^{2}=\left\|\widetilde{D} z^{n}\right\|_{\operatorname{Mult}\left(\mathcal{H}_{f}\right)}^{2} \leq\|\widetilde{D}\|^{2}\left\|z^{n}\right\|_{\operatorname{Mult}\left(\mathcal{H}_{f}\right)}^{2}=\|\widetilde{D}\|^{2} \frac{1}{a_{n}} \text {. }
$$

Thus, (iii) holds.

It is not hard to modify Example 6.12 in [3] to see that the conditions in the preceding lemma are not always satisfied.

Now recall that for $s \in \mathbb{R}, \mathcal{H}_{s}$ is the reproducing kernel Hilbert space on the unit disc with kernel

$$
k(z, w)=\sum_{n=0}^{\infty}(n+1)^{s}(z \bar{w})^{n}
$$

Corollary Let $\rho$ be a character of $\operatorname{Mult}\left(\mathcal{H}_{s}\right), s \leq 0$. If $\lambda \in \mathbb{D}$, and $\rho(z)=\lambda$, then $\rho(f)=f(\lambda)$ for every $f \in \operatorname{Mult}\left(\mathcal{H}_{s}\right)$. For $s<-1$, the same is true for $\lambda \in \mathbb{T}$.

Proof Case: $\lambda \in \mathbb{D}, s \leq 0$. Since Mult $\mathcal{H}_{s}$ is automorphism invariant, it is enough to prove this for $\lambda=0$. Let $f \in \operatorname{Mult}\left(\mathcal{H}_{s}\right)$ be such that $f(0)=0$, i.e., $f \in \operatorname{ker} \rho_{0}$. By the above lemma, $f=z g$, where $g \in \operatorname{Mult}\left(\mathcal{H}_{s}\right)$. But then $\rho(f)=\rho(z) \rho(g)=0$, so $f \in \operatorname{ker} \rho$. We find that the maximal ideal $\operatorname{ker} \rho$ contains the maximal ideal ker $\rho_{0}$, thus $\rho=\rho_{0}$. 
Case: $\lambda \in \overline{\mathbb{D}}, s<-1$. When $s<-1$ the argument from the previous paragraph does not suffice, since automorphisms of the disc do not take 0 to $\mathbb{T}$. We use some results from Shields [4, Section 9]. He provides a sufficient condition (Prop.32) for the multiplier algebra to be strictly cyclic, which is readily verified for $\mathcal{H}_{s}$ if $s<-1$. Then he shows (Prop.31, Cor.1) that the maximal ideal space of $\operatorname{Mult}\left(\mathcal{H}_{s}\right)$ is naturally identified with $\overline{\mathbb{D}}$.

2.2 Proof that $F$ is a Biholomorphism in the Case that $\mathcal{M}_{V_{1}}$ Satisfies (1) and (2) and $\varphi$ is an Isomorphism

Assume that $\varphi$ is an isomorphism. If $\mathcal{M}_{V_{1}}$ satisfies (1) and (2), then the proof of Theorem 2.4 shows that $\varphi^{*}$ takes point evaluations to point evaluations, that $F$ is holomorphic, and that $\varphi$ is given by

$$
\varphi(h)=h \circ F .
$$

To show that $F$ is biholomorphism, one needs to apply the same arguments to $\left(\varphi^{-1}\right)^{*}$, but for this one needs that $\left(\varphi^{-1}\right)^{*}$ takes point evaluations to point evaluations. This is achieved as follows.

By an adaptation of [2, Section 11.3], the fact that $\varphi$ is implemented by composition implies that $\varphi$ is weak-* continuous. Since the closed unit ball $B_{1}$ of $\mathcal{M}_{V_{1}}$ is weak-* compact, and since the weak- $*$ topology on $\mathcal{M}_{V_{2}}$ is Hausdorff, $\left.\varphi\right|_{B_{1}}: B_{1} \rightarrow \varphi\left(B_{1}\right)$ is a homeomorphism in the weak-* topologies. Every bounded set in $\mathcal{M}_{V_{2}}$ is contained in $r \varphi\left(B_{1}\right)$ for some $r>0$, hence $\varphi^{-1}$ is weak- $*$ continuous on bounded sets. It follows from the Krein-Smulian theorem that $\varphi^{-1}$ is weak- $*$ continuous. In particular, $\left(\varphi^{-1}\right)^{*}$ takes point evaluations to point evaluations, and the proof of Theorem 2.4 can be completed as in the paper.

\section{References}

1. Davidson, K., Pitts, D.: The algebraic structure of non-commutative analytic Toeplitz algebras. Math. Ann. 311, 275-303 (1998)

2. Davidson, K., Ramsey, C., Shalit, O.: The isomorphism problem for some universal operator algebras. Adv. Math. 228, 167-218 (2011)

3. Davidson, K., Ramsey, C., Shalit, O.: Operator algebras for analytic varieties. Trans. Amer. Math. Soc. (to appear). arXiv:1201.4072v4 [math.OA]

4. Shields, A.L.: Weighted shift operators and analytic function theory. In: Topics in Operator Theory. Mathematical Surveys, vol. 13. American Mathematical Society, Providence, pp. 49-128 (1974) 